\title{
Melanoma leptomeningeal dissemination following frontoparietal metastasis surgery: Case report and review of the literature
}

\author{
MARÍA SERENO MOYANO ${ }^{1 *}$, GERARDO GUTIÉRREZ-GUTIÉRREZ ${ }^{2 *}$, ISABEL RODRÍGUEZ-ESTEBAN ${ }^{3}$, \\ GEMMA SÁNCHEZ CRESPO ${ }^{4}$ and ENRIQUE CASADO ${ }^{1}$
}

\author{
Departments of ${ }^{1}$ Medical Oncology, ${ }^{2}$ Neurology, ${ }^{3}$ Pathology, and ${ }^{4}$ Clinical Analysis, \\ Infanta Sofía Hospital, San Sebastián de los Reyes, Madrid, Spain
}

Received August 2, 2010; Accepted September 20, 2010

DOI: 10.3892/ol.2010.194

\begin{abstract}
We present the case of a patient with a solitary left frontoparietal brain metastasis of melanoma previously treated with surgery. Three months later, the patient was admitted to the emergency room in a confusional state with meningeal signs. A cerebrospinal fluid (CSF) test and magnetic resonance imaging findings suggested a subarachnoid haemorrhage (SAH) and/or meningeal carcinomatosis. The results of a cytological examination of the CSF showed neoplastic epithelial cells consistent with metastatic melanoma cells. Resection of metastatic posterior fossa lesions is often cited as a risk factor for leptomeningeal dissemination, however, when the resection is limited to the anterior fossa, this complication is relatively rare. In contrast, SAH may be a complication of leptomeningeal dissemination and responsible for acute meningeal syndrome. Treatment with high doses of corticoids did not show any improvement, and intrathecal chemotherapy was not possible due to the patient's poor functional status. She succumbed 1 week after admission.
\end{abstract}

\section{Introduction}

Melanoma is one of the most common tumors to metastasize to the brain. Leptomeningeal carcinomatosis (LMC) is defined as a malignant infiltration of the pia mater and the arachnoid membranes. LMC is one of the most serious complications

Correspondence to: Dr María Sereno Moyano, Department of Medical Oncology, Infanta Sofía Hospital, C/Julio Rey Pastor 6, portal 6, 4b, 28702 San Sebastián de los Reyes, Madrid, Spain

E-mail: mariasereno75@gmail.com

Dr Gerardo Gutiérrez-Gutiérrez, Department of Neurology, Infanta Sofía Hospital, C/Julio Rey Pastor 6, portal 6, 4b, 28702 San Sebastián de los Reyes, Madrid, Spain

E-mail: g3.neuro@gmail.com

*Contributed equally

Key words: leptomeningeal carcinomatosis, subarachnoid haemorrhage, complications of brain metastasis surgery occurring in cancer patients. According to the results of an extensive autopsy study, the incidence of LMC was 5-8\% in cancer patients (1). Since a significant proportion of these patients exhibited asymptomatic microscopic disease, the clinical diagnosis of LMC is established in only 2-4\% of the patients diagnosed with LMC at autopsy. LMC is frequently detected in patients with leukaemia, breast cancer, lymphoma, melanoma and lung cancer. Among solid tumors, LMC is observed more frequently in cases of disseminated and progressive disease. Although a subset of patients, particularly those with lymphoma or breast cancer, may survive for more than 12 months with a reasonable quality of life, leptomeningeal metastasis from solid tumors such as melanoma, is associated with a poor overall prognosis. The treatment of LMC is palliative and unsatisfactory. At present, intrathecal chemotherapy is the standard treatment, but this procedure does not demonstrate a significant increase in survival, and at best is only palliative (2).

\section{Case report}

We present a case of a 44-year-old female who was diagnosed with cutaneous melanoma on the back in August 2008. She underwent surgery, and sentinel node testing was negative. Pathological findings were consistent with nodular melanoma, Clark III, Breslow thickness $2.62 \mathrm{~mm}, 9$ mitoses/field, nonulcerated and without vascular or perineural invasion. No satellite lesions were found upon pathological examination. The work-up did not show distant metastases (physical examination, abdominal ultrasound and chest X-ray). Following surgery, the patient was referred to the Medical Oncology Department to evaluate whether adjuvant treatment with interferon was required. The lesion did not show poor prognostic criteria (Clark <IV, non-ulcerated, non-lymphovascular invasion); therefore, she did not receive adjuvant interferon treatment according to Kirkwood's regimen (3). The patient continued with routine follow-up every 3 months including a physical examination, abdominal ultrasound, chest X-ray and blood test with hemogram and basic biochemistry that included lactate dehydrogenase and transaminases.

In June 2009, she was admitted to the emergency room with a persistent headache, predominantly in the frontal area, that did not respond to anti-inflammatory drugs. A head 
a

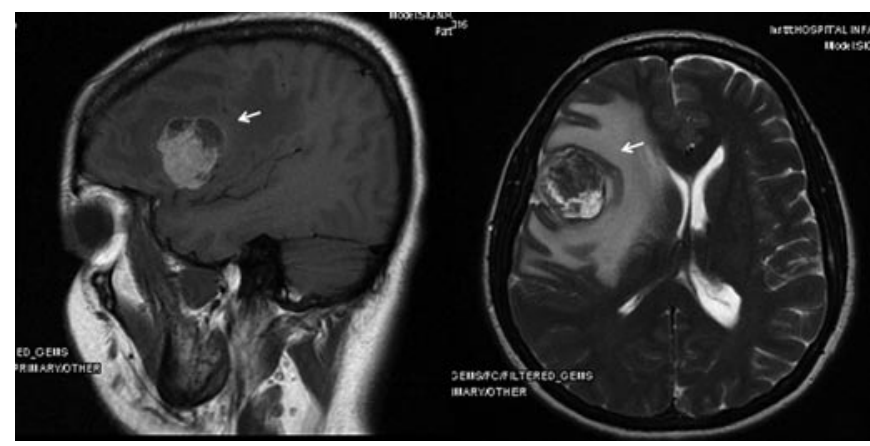

Figure 1. (a and b) MRI of the brain showed a 28-mm sized lesion located in the right frontoparietal area with perilesional edema with subfalcial hernia and compression of the frontal horn of the right lateral ventricle. All images were suggestive of metastasis.

computed tomography (CT) and magnetic resonance imaging (MRI) showed a 28-mm lesion located in the right frontoparietal area with considerable perilesional edema with subfalcial hernia and compression of the frontal horn of the right lateral ventricle. All images were suggestive of metastasis. An MRI of the brain confirmed the CT scan findings and did not show additional lesions in the cerebral parenchyma (Fig. 1a and b). Steroid therapy was commenced (dexamethasone $8 \mathrm{mg}$ tid), and after 1 day of steroid treatment, the headache almost subsided. The patient underwent a work-up study with body CT scan and PET-TAC. In all of these studies, no evidence of extra-cerebral disease was found. The patient was referred to the Neurosurgical Department to consider resection of the solitary melanoma brain metastasis.

In September 2009, she underwent resection for brain metastasis. Pathological examination showed features suggestive of melanoma metastasis with a resection margin free of tumor. To reduce the risk of brain dissemination, the patient received whole brain radiation 15 days after resection.

The patient continued with the routine follow-up, including body CT and MRI of the brain every 3 months. A PET-TAC carried out on October 2009 did not show any uptakes suggestive of malignant disease.

In December 2009, she was admitted to the emergency room because of an acute confusional state and severe back pain. The condition was extremely acute and progressive. The pain did not subside with anti-inflammatory drugs. A fever of $38^{\circ} \mathrm{C}$, blood pressure 150/110, somnolence (Glasgow Coma Scale 13) and positivity for meningeal signs were noted. Blood testing did not show relevant findings. A non-traumatic lumbar puncture was performed. The macroscopical appearance of the cerebrospinal fluid (CSF) was haematic and cloudy, and the opening pressure was $40 \mathrm{mmHg}$. The biochemical analysis of the CSF showed a glucose of $6 \mathrm{mg} / \mathrm{dl}$ (40-70), proteins $265.8 \mathrm{mg} / \mathrm{dl}(15-40)$ and lactate 7.1 (0-3). The cell consisted of leucocytes 38 cells $/ \mathrm{mm}^{3}$; neutrophils $2 \%$; lymphocytes $98 \%$ and erythrocytes, 1,000 cells $/ \mathrm{mm}^{3}$. CT of the head showed post-surgical changes and possible bleeding (Fig. 2a and b). The MRI showed a right frontal area of malacia related to the previous metastasis surgery with 19x13 mm of transversal and anteroposterior diameters. Various changes surrounding this area were observed, suggestive of post-radiotherapy gliosis. In

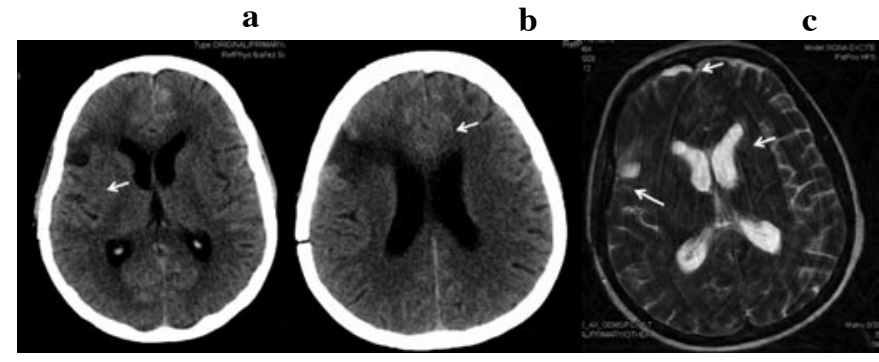

Figure 2. (a and b) CT of the brain showed post-surgical changes and possible post-resection bleeding. (c) The MRI findings showed a right frontal area of malacia related to the previous metastasis surgery with $19 \times 13 \mathrm{~mm}$ of transversal and anteroposterior diameters with surrounding changes suggestive of post-radiotherapy gliosis. In T1-weighted image (T1WI) in this malacic area a hyperintensity, as well as sulcus hyperintensity, possibly related to haemorrhage and/or meningeal carcinomatous were noted. The third ventricle was enlarged with periventricular hyperintensity and acute hydrocephaly.
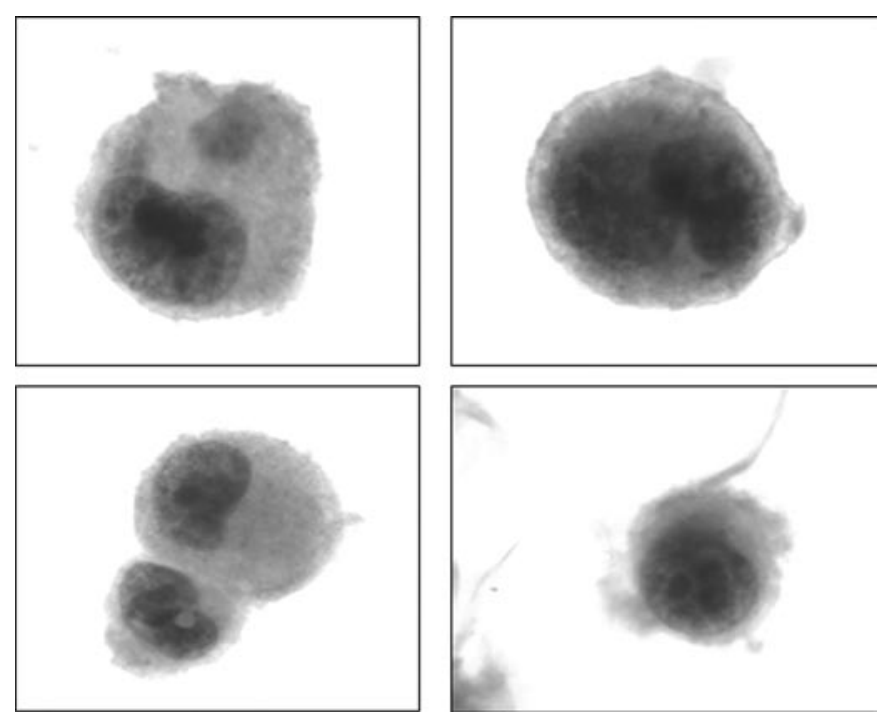

Figure 3. Images of the cytology results of CSF in liquid media (ThinPrep ${ }^{\circledR}$ ) with Papanicolaou staining; magnification, x63. This image showed a CSF extension with lymphocytosis and isolated enlarged cells, uninucleated or binucleated with a number of prominent nucleoli without nuclear pseudoinclusions; suspect of malignancy and morphologically consistent with melanoma.

T1-weighted images (T1WI) in this malacic area a hyperintensity was observed that was potentially related to haemorrhage. In convexity, in the posterior parasagital region and particularly in the frontal area, a sulcus hyperintensity was described. The findings were suggestive of a subarachnoid haemorrhage (SAH) and/or meningeal carcinomatosis. The third ventricle was enlarged with periventricular hyperintensity consistent with early acute hydrocephaly (Fig. 2c). Interpretation of these findings by radiologists was difficult due to the severe agitation of the patient even after administration of intensive sedative medications.

Based on the MRI images a possible diagnosis was SAH and/or meningeal infiltration. The cytological examination of the CSF showed neoplastic epithelial cells consistent with metastatic melanoma cells. Immunohistochemical analysis was not performed due to the small number of atypical cells found (Fig. 3). Treatment of high doses of corticoids did not 
Table I. Patient survival when one or more treatments were administered for brain metastases. ${ }^{\text {a }}$

\begin{tabular}{lrrc}
\hline Treatment & No. & $\%$ & $\begin{array}{c}\text { Median survival } \\
\text { (months) }\end{array}$ \\
\hline None & 83 & 23.3 & 2.04 \\
WBRT alone & 100 & 28.2 & 3.98 \\
RS alone & 26 & 7.3 & 9.87 \\
Surgery alone & 36 & 10.1 & 8.16 \\
WBRT + RS & 20 & 5.6 & 9.44 \\
Surgery + WBRT & 58 & 16.3 & 8.81 \\
Surgery + RS & 20 & 5.6 & 13.75 \\
Surgery + WBRT + RS & 12 & 3.4 & 10.20 \\
\hline
\end{tabular}

a Data from Raizer et al (4). WBRT, whole-brain radiation; RS, radiosurgery.

show any improvement, and the patient developed a progressive confusional syndrome. Intrathecal chemotherapy was not possible due to her poor functional status. The patient succumbed 1 week after admission.

\section{Discussion}

Whereas brain metastasis can be a complication associated with various types of solid tumors, such as small-cell lung cancer and melanoma, leptomeningeal dissemination (LMD) is a relatively infrequent complication of solid tumors. Again, the most common solid malignancies associated with brain metastasis are breast cancer, small-cell lung cancer and melanoma. The majority of patients with leptomeningeal metastasis have advanced disease, and it rarely occurs as an initial or unique presentation.

We presented a case of a patient with a solitary brain metastasis of melanoma previously treated with surgery. Numerous trials have served as level I evidence supporting the role of surgery in patients with single brain metastasis, particularly for recursive partitioning analysis class I. Some authors support the combination of surgery followed by radiosurgery and whole-brain radiation as one of the treatment modalities that achieves the longest progression-free survival (4) (Table I). However, in our patient, resection was followed by whole-brain radiation instead of whole-brain radiation alone (5). Surgery of brain metastases is, however, not free of complications. At present, there is discussion whether surgery for brain metastasis causes meningeal dissemination. Resection of metastatic posterior fossa lesions (MPLFs) is often cited as a risk factor for LMD, but a review of the literature suggests the need for further evidence (6-8).

One recent publication demonstrated the risk of meningeal dissemination with resection of MPLFs compared to radiosurgery. Suki et al found an increased risk of LMD in patients treated with surgery compared to patients treated with radiosurgery, although the difference did not reach statistical significance (9). The slow CSF flow in the posterior fossa region of the brain that promotes the deposition of circulating cells, and the subarachnoid space and cisterna magna in this region, which may offer a nidus for malignant cells, may increase the risk of LMD following resection of tumors in this region (10). Conversely, the absence of such a CSF-containing space over the hemispheres may explain the low incidence of LMD in cases of supratentorial lesions. There is no formal estimate of the minimum number of tumor cells in the CSF that are sufficient to cause LMD. Extrapolating from animal models, in which the introduction of 3,000 tumor cells has resulted in rapid death from LMD, the number is likely to be relatively small (11). Van der Ree et al published a review of the records of all the patients operated on for brain metastasis between January 1990 and August 1995 in their centre. The authors included 28 patients with melanoma brain metastasis who underwent surgery for intracranial lesions. Their results showed that 9 patients (33\%) developed meningeal metastasis 2-13 months after surgery, which included 6 of the 9 patients operated on for posterior fossa metastasis (12). In contrast, DeAngelis et al found that $38 \%$ of patients developed leptomeningeal metastasis following surgery for cerebellar metastasis, while only $4.7 \%$ of the patients were treated for a supratentorial metastasis (13).

The present case report described a patient who developed LMC 3 months after solitary brain resection in the anterior fossa. She also presented with meningeal signs, and an MRI showed images suggestive of SAH and/or LMC. The CFS analysis showed malignant as well as red blood cells. It is well known that melanoma meningeal lesions bleed, explaining the association between LMC and SAH. The relationship between SAH and leptomeningeal metastases was previously reported in only 5 cases in the literature. The diagnosis of SAH secondary to neoplastic seeding is based on CSF studies and on neuroimaging evaluations, which are confirmed by autopsy or surgery (14). In a retrospective evaluation of 120 patients treated for leptomeningeal metastases, Lossos et al reported that 3 patients had spontaneous SAH in the absence of a bleeding tendency. Resection of an intraparenchymal posterior fossa tumor antedated the development of subarachnoid seeding in 3 of the 5 patients (15).

Taking into account the radiological findings, the CSF examination and the previous surgery for melanoma brain metastasis, we concluded that our patient presented with an SAH secondary to LMC. We attributed this complication to LMC since the MRI did not show aneurisms or any other type of malformation. The patient outcome was unforeseen, particularly when we consider that the PET-TAC did not show any uptakes suggestive of cancer in other locations. Although the risk of LMC was low following brain metastasis resection due to its location, we suggest that there was a clear relationship between the surgery and LMC. In addition, this patient suffered an acute meningeal syndrome which may have been secondary to the SAH according to the haemorrhagic CSF and MRI findings. This complication, as is well known, is not a rare consequence of melanoma meningeal dissemination.

\section{References}

1. Pentheroudakis $\mathrm{G}$ and Pavlidis N: Management of leptomeningeal malignancy. Expert Opin Pharmacother 6: 1115-1125, 2005 . 
2. Bruno MK and Raizer J: Leptomeningeal metastases from solid tumours (meningeal carcinomatosis). Cancer Treat Res 125: $31-52,2005$.

3. Tarhini AA and Kirkwood JM: Clinical and immunologic basis of interferon therapy in melanoma. Ann NY Acad Sci 1182: 47-57, 2009.

4. Raizer J, Hwu W, Panageas K, et al: Brain and leptomeningeal metastases from cutaneous melanoma: survival outcomes based on clinical features. Neuro Oncol 10: 199-207, 2008.

5. Sloan A, Nock C, Estein D, et al: Diagnosis and treatment of melanoma brain metastasis: a literature review. Cancer Control 3: 248-254, 2009.

6. Chamberlain MC and Johnston SK: Neoplastic meningitis: survival as a function of cerebrospinal fluid cytology. Cancer 115 1941-1946, 2009

7. Vellin JF, Achim V, Sinardet D, et al: Rapidly developing leptomeningeal carcinomatosis following anterior skull base surgery: a case report. Auris Nasus Larynx 34: 565-567, 2007.

8. Yu H, Mitsumori M, Nagata Y, et al: Meningeal carcinomatosis in patients with breast cancer: report of 8 patients. Breast Cancer 8: 74-78, 2001.

9. Suki D, Abouassi H, Patel A, et al: Comparative risk of leptomeningeal disease after resection or stereotactic radiosurgery for solid tumor metastasis to the posterior fossa. J Neurosurg 108: 248-257, 2008.
10. Grossman SA and Krabak MJ: Leptomeningeal carcinomatosis. Cancer Treat Rev 25: 103-119, 1999.

11. Norris LK, Grossman SA and Olivi A: Neoplastic meningitis following surgical resection of isolated cerebellar metastasis: a potentially preventable complication. J Neurooncol 32: 215-233, 1997.

12. Van der Ree T, Dippel D, Avezaart C, Sillevis Smitt PA, Vecht CJ and van den Bent MJ: Leptomeningeal metastasis after surgical resection of brain metastases. J Neurol Neurosurg Psychiatry 66: 225-227, 1999.

13. De Angelis LM, Mandell LR, Thaler HT, et al: The role of postoperative radiotherapy after resection of single brain metastasis. Neurosurgery 24: 798-805, 1989.

14. Chang C, Kuwana $\mathrm{N}$ and Ito S: Spinal leptomeningeal metastases of giant cell glioblastoma associated with subarachnoid haemorrhage: case report. J Clin Neurosci 8: 56-59, 1998.

15. Lossos A and Siegal T: Spinal subarachnoid haemorrhage associated with leptomeningeal metastases. J Neurooncol 12: 167-171, 1992. 